\title{
Shot-to-Shot and Long-Term CEP-Stable Front-End for a Parallel Optical Waveform Synthesizer
}

\author{
Roland E. Mainz, ${ }^{1,2, *}$ Giulio Maria Rossi, ${ }^{1,2,}$ ' Giovanni Cirmi, ${ }^{1,2}$ Yudong Yang, ${ }^{1,2}$ \\ Oliver D. Mücke, ${ }^{1,2}$ and Franz X. Kärtner ${ }^{1,2}$ \\ ${ }^{1}$ Center for Free-Electron Laser Science, Deutsches Elektronen-Synchrotron DESY, Notkestraße 85, 22607 Hamburg, Germany \\ ${ }^{2}$ Physics Department and The Hamburg Center for Ultrafast Imaging, University of Hamburg, \\ Luruper Chaussee 149, 22761 Hamburg, Germany \\ *roland.mainz@cfel.de
}

We present a highly automated front-end of a Ti:Sa-based optical waveform synthesizer capable of driving multiple supercontinua with stable and controlled carrier-envelope phases. The remarkable CEP-stability of $217 \mathrm{mrad}$ rms singleshot will allow shot-to-shot and long-term stable waveform synthesis.

OCIS codes: (320.7100) Ultrafast measurements;

(190.4970) Parametric oscillators and amplifiers.

\section{INTRODUCTION}

Driven by the goal to investigate the response of matter to arbitrarily sculpted electromagnetic field transients, the realization of a waveform synthesizer in the optical region is an extremely challenging task. One of the promising approaches to realize such a device is the combination of multiple parallel optical parametric (chirped-pulse) amplifier (OP(CP)A) outputs, that can be tuned to cover different spectral regions, allowing for an overall bandwidth of more than two octaves [1]. In former works [2] we envisaged the possibility to seed each of the different $\mathrm{OP}(\mathrm{CP}) \mathrm{A}$ channels with its own white-light supercontinua (WLs), in contrast to an earlier approach, where a single supercontinuum is split via dichroic mirrors, each of its separate spectral regions is then seeding a different $\mathrm{OP}(\mathrm{CP}) \mathrm{A}$ channel. The multi-WL approach offers significant advantages such as the possibility of implementing different crystal materials/thicknesses and optimizing focusing geometries for each WL, in order to obtain a higher phase and energy stability in the specific spectral region that is intended to be used as seed. Moreover, in a single WL seeding scheme the supercontinuum needs (for space constraints reasons) to propagate for a longer distance before being split and reaching the different $\mathrm{OP}(\mathrm{CP}) \mathrm{A}$ channels. The ultra-broadband nature and the complex spatial profile of a WL output make it difficult to propagate long distances while avoiding unfavorable changes in the beam profile and dispersion. On the other hand, the generation of multiple WLs raises questions about the level of coherence

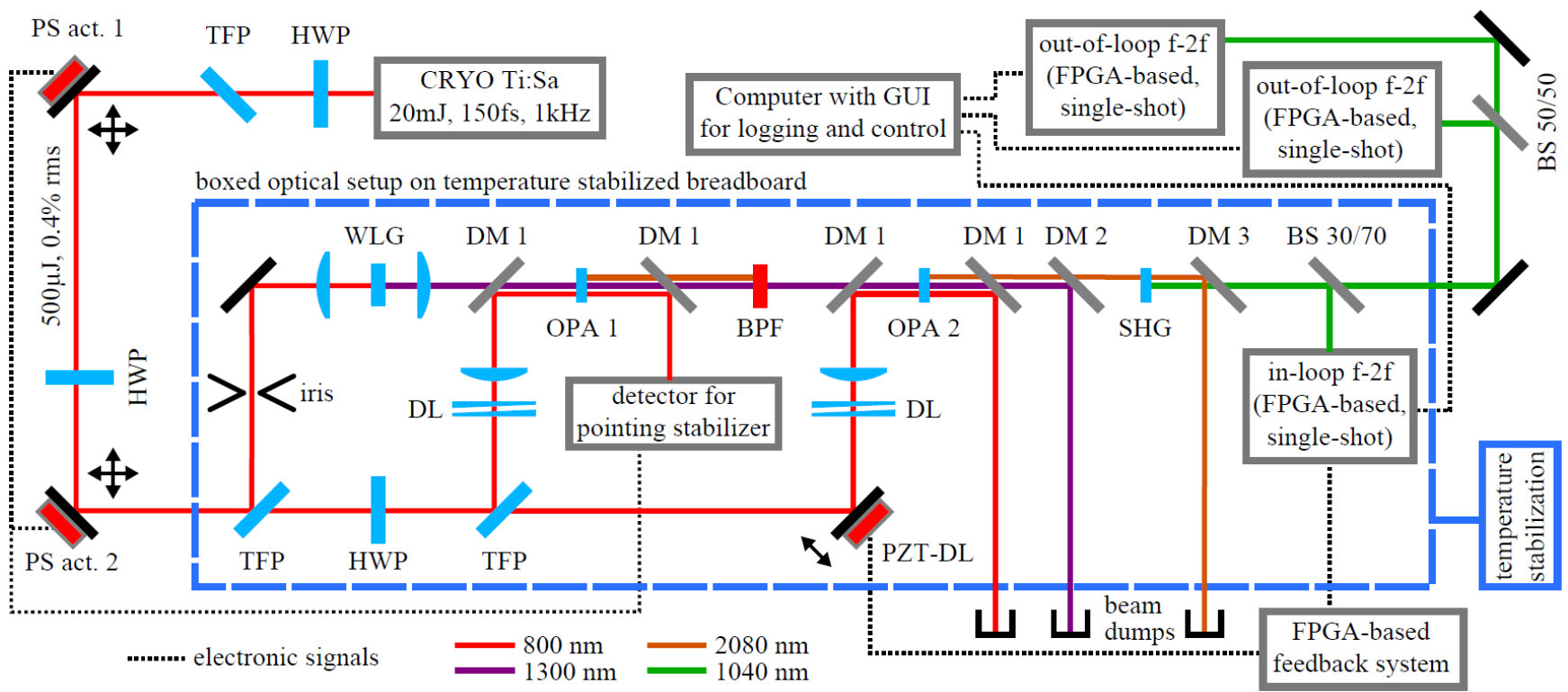

Fig. 1. Scheme of the setup for the generation of passively CEP-stable pulses at $1040 \mathrm{~nm}$ from a non-CEP-stabilized Ti:sapphire laser system. The driving laser $(800 \mathrm{~nm}, 150 \mathrm{fs})$ is pointing stabilized (PS) and split into several portions on a boxed and temperature stabilized breadboard. A first portion is driving a WL, whose region around $1300 \mathrm{~nm}$ is amplified by a first OPA crystal (OPA 1, BBO Type II, 20, $2.5 \mathrm{~mm}$ ). The custom-designed dichroic mirrors DM 1 are used to combine/separate the pump beam before/after the OPAs. A band-pass filter (BPF) transmits a 12.5-nm wide region around $1300 \mathrm{~nm}$ from the signal (preventing double seeding), which is then used to seed a second OPA (OPA 2, BBO Type II, $20^{\circ}, 3.5 \mathrm{~mm}$ ), which generates a passively CEP-stabilized idler beam at 2080 $\mathrm{nm}$. DM 2 filters out the signal and the transmitted idler is frequency-doubled in a $0.5 \mathrm{~mm}$ thick BBO (SHG). DM 3 removes the fundamental and beam splitters (BS) divide the output at $1040 \mathrm{~nm}$ into three portions, each used to drive a WL. The CEP of each WL is detected by means of three $\mathrm{f}-2 \mathrm{f}$ interferometers. The $\mathrm{f}$ $2 \mathrm{f}$ setups double (in a BBO) the IR-part of the WL spectrum and beat it with its VIS part on a single-shot spectrometer. An FPGA-based processing unit calculate (within $100 \mu \mathrm{s}$ ) the FFT of the in-loop f-2f interference pattern. Then the phase of the strongest spectral component is unwrapped and fed to a PIcontroller that drives a piezo which delays the pump of the second OPA (PZT-DL). The setup is controlled by a PC and all three f-2f spectra are recorded in a synchronized fashion. 
with respect to each other, a crucial parameter for a parallel waveform synthesizer.

\section{CEP-STABLE FRONT-END}

Here we present a highly automated OPA-based, CEP-stable and CEP-controlled, front-end of a Ti:sapphire-driven waveform synthesizer, consisting of up to four independently driven CEP-stable WLs, suitable for seeding multiple $\mathrm{OP}(\mathrm{CP}) \mathrm{A}$ channels (see Fig. 1). The basic CEP-stabilization principle relies on the well-known passive stabilization of the idler produced by difference-frequency generation in a WL seeded OPA. In order to obtain the lowest energy fluctuations possible, the phase matching condition chosen such that the group velocity of the pump pulse stands in between the idler and signal group velocities. This configuration allows for high conversion efficiency and low energy fluctuations, since the pump reaches depletion [3]. The final output consists of $56 \mu \mathrm{J}$ setup to perform an in-loop f-2f measurement for retrieving the CEP by driving a WL in a YAG crystal. The IR part of the WL-spectrum is frequency doubled in a $\mathrm{BBO}$ to beat with the VIS part on a single-shot spectrometer. A home-made FPGAbased feedback system is actively stabilizing and controlling the CEP by a piezo-actuator, which changes the delay between pump and seed in the second OPA. Outside of the setup are two virtually identical out-of-loop f- $2 \mathrm{f}$ setups equipped with single-shot spectrometers as well. This unique configuration allows us to both validate the in-loop measurement, and determine an upper limit for the CEP noise among the different WLs.

In order to achieve the best possible shot-to-shot and longterm stability, a number of expedients were implemented: the breadboard was boxed and temperature stabilized by heating it $1 \mathrm{~K}$ above room temperature, achieving few $\mathrm{mK}$ rms temperature stability; the optical beam path was kept as short

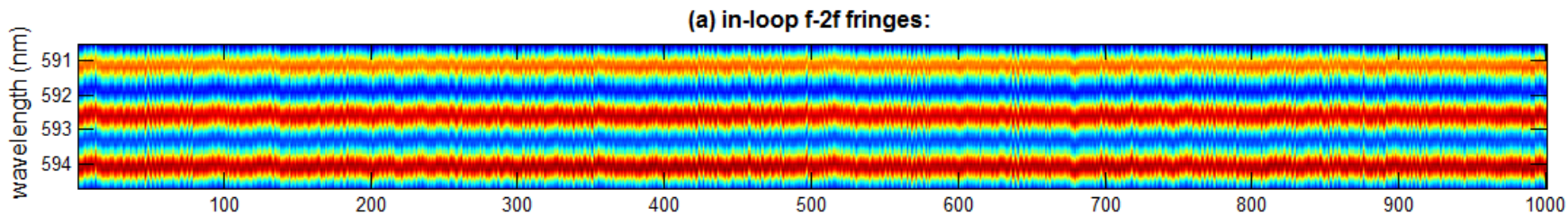

(b) primary out-of-loop f-2f fringes:

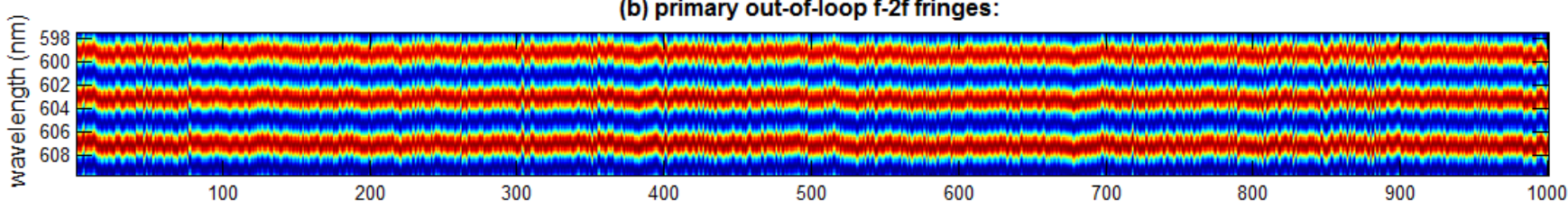

(c) secondary out-of-loop f-2f fringes:

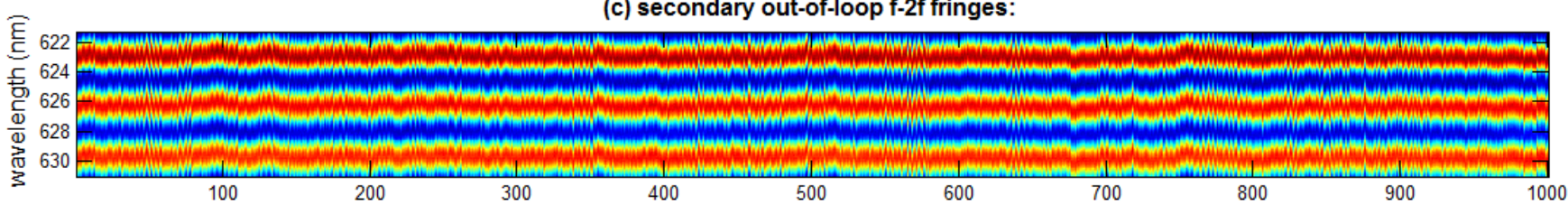

(d) retrieved phases of all f-2f devices:

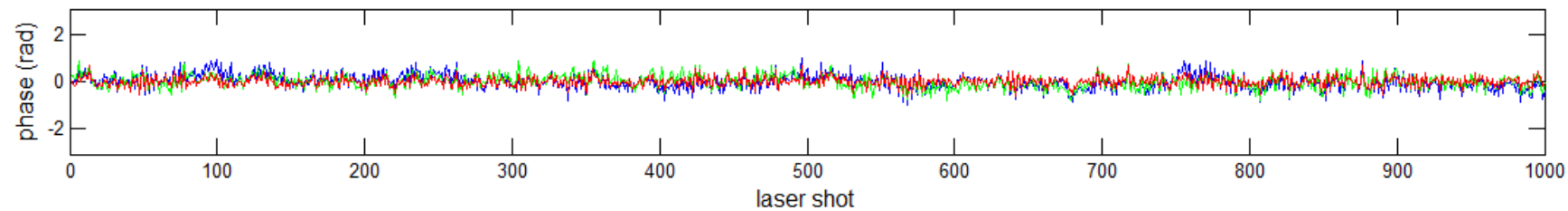

Fig. 2. (a) The single-shot and every-shot $\mathrm{f}-2 \mathrm{f}$ spectral fringe phase is retrieved and the CEP is actively stabilized by a low-latency FPGA-based active stabilization and control unit (in-loop f-2f). (b-c) The two almost identical f-2f setups are used to verify the quality of the CEP-lock and estimate the measurement noise of an individual f-2f setup. (d) The retrieved CEP of the three $\mathrm{f}-2 \mathrm{f}$ setups are plotted (red in-loop, blue/green out-of-loop). The phase noise is $217 \mathrm{mrad}$ in-loop and $280 \mathrm{mrad}$ out-of-loop each. The difference of the two out-of-loop phases yields $256 \mathrm{mrad}$, leaving a measurement error of $180 \mathrm{mrad}(256 / \mathrm{sqrt}(2))$ for the $\mathrm{f}-2 \mathrm{f}$. The pure out-of-loop CEP-noise can be estimated to be about $200 \mathrm{mrad}\left(\mathrm{sqrt}\left(280^{\wedge} 2-180^{\wedge} 2\right)\right)$.

of non-CEP-stable signal pulses at $1300 \mathrm{~nm}$ and $35 \mu \mathrm{J}$ of passively CEP-stable idler pulses at $2080 \mathrm{~nm}$. The CEP-stable idler pulses are then frequency-doubled in a $0.5 \mathrm{~mm}$ Type I $\mathrm{BBO}$, yielding $10 \mu \mathrm{J}$ of CEP-stable pulses at $1040 \mathrm{~nm}$ with a pulse duration of $85 \mathrm{fs}$, nearly transform limited (the SHG conversion efficiency is intentionally kept low in favor of beam quality). Those pulses are suitable for WL generation in the 500-2500 $\mathrm{nm}$ region. The $1040 \mathrm{~nm}$ pulses are split in nearly three equal portions and one is used inside the boxed as possible, by means of custom designed dichroic mirrors, that transmit the signal through the whole setup avoiding reflections, thus undesired vibrations; an home-build piezo actuator was integrated in the second stage pump-path in order to further stabilize and control the CEP of the idler pulses. Moreover, a low-latency FPGA-based spectrometer capable of single-shot and every-shot acquisition was developed in-house in order to fully characterize the CEP-stability and achieve the most effective active stabilization. At last a commercial 
pointing stabilizer (TEM-Messtechnik), referenced to the OPA breadboard was used to avoid any possible misalignment of the input laser-beam.

\section{PERFORMANCE CHARACTERIZATION}

The CEP-stable output consists in $35 \mu \mathrm{J}$ centered at $2080 \mathrm{~nm}$ (with fluctuations of $0.5 \% \mathrm{rms}$ over $1000 \mathrm{sec}$ ) and after doubling about $10 \mu \mathrm{J}$ centered at $1040 \mathrm{~nm}$, with energy stability better than $1 \%$ rms over $1000 \mathrm{sec}$ (see Fig. 3). The $1040 \mathrm{~nm}$ pulse duration was characterized by SHG-FROG to be $85 \mathrm{fs}$ and transform-limited. The pulses generated are remarkably CEP-stable as well. The in-loop f-2f shows singleshot and every-shot CEP fluctuations of $217 \mathrm{mrad}$ (see Fig. 3, this reduces to $114 \mathrm{mrad}$ when averaging over 4 shots, or 17 mrad averaging over 100 shots), while the two out-of-loop CEP-measurements exhibit a stability of 270 and $290 \mathrm{mrad}$, respectively (see Fig. 2). Most of the difference can be attributed to air fluctuations and slow drifts, since the out-ofloop $\mathrm{f}-2 \mathrm{f}$ setups are placed outside the temperature-stabilized after the subtraction). The setup has been running for more than a week without any noticeable drifts. A 15 minutes dataset (Fig. 3(a)) has been analyzed: the CEP is mainly below $250 \mathrm{mrad}$ rms (averaged over 1000 shots) and the few events where it jumps are attributed to the action of the stepper motors of the pointing stabilizer (this will be fixed by moving the two actuators more far away from each other). Both the energy and the CEP shows no slow drifts, being always close to their mean. Longer measurements will soon be analyzed.

Moreover, in contrast to [4], we observed a direct relation between "interferometric stability" and CEP-stability, meaning that an elongation of the pump path by one seed wavelength (1300 $\mathrm{nm}$ in our experiment) corresponds roughly to a $2 \pi$ change of the CEPs of the signal and idler pulses (see Fig. 3).

To explain this behavior we consider the following simple model, where the pump pulse is described by an envelope and a carrier:

$$
E_{\text {pump }}(t)=A_{\text {pump }}(t) \exp \left(-i\left(\omega_{\text {pump }} t+\varphi_{\text {pump }}\right)\right)
$$

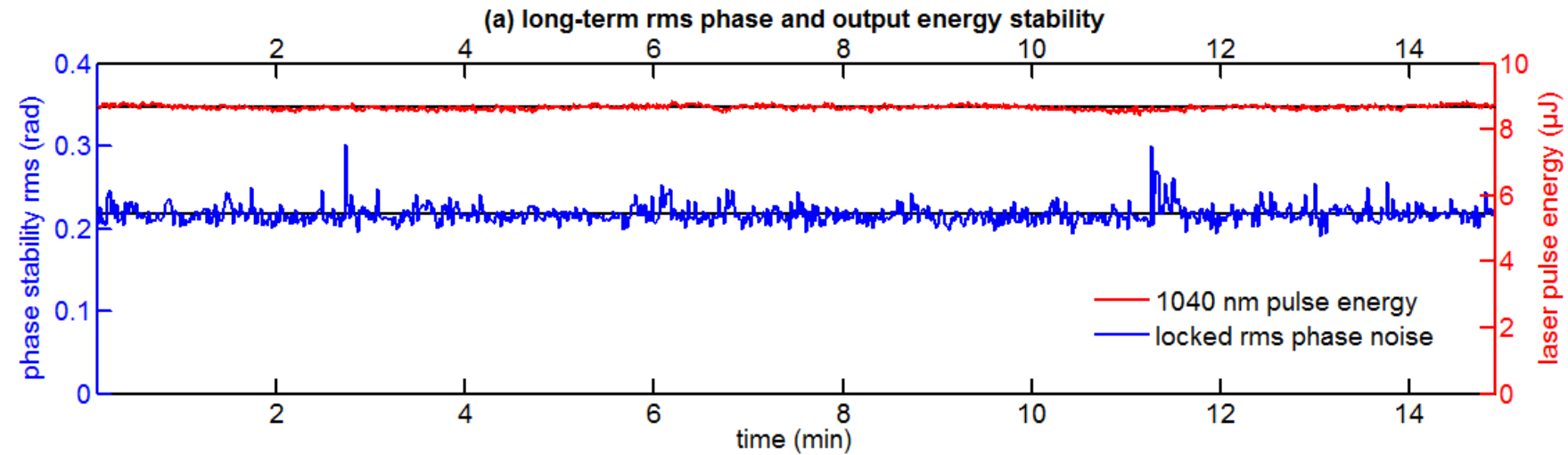

(b) IL and OOL1/2 phase towards piezo induced delay

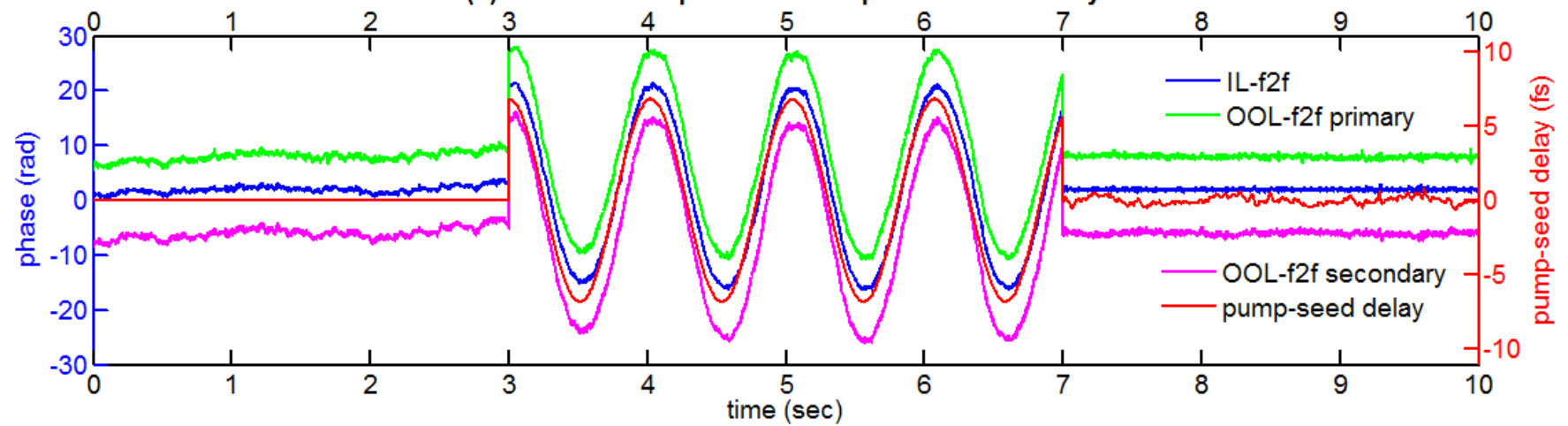

Fig. 3. (a) A typical 15 minutes long in-loop CEP noise trace (blue line, every points is the phase rms over 1k shots) and its 15 min average (black line). Plotted in red, the single-shot pulse energy and its 15 min average (black line). (b) The three single-shot measurement traces of the CEP are shown over a period of $10 \mathrm{sec}$, at first unlocked, then modulated by the piezo actuator, finally locked to a fixed value. The piezo actuator introduces a sinusoidal pumpseed delay in the second OPA with a period of $1 \mathrm{~Hz}$ and an amplitude of $13.6 \mathrm{fs}$ peak-to-peak. According to the simple model described below this delay should correspond to a CEP change of $39.4 \mathrm{rad}$, and the measured change is $36.5 \mathrm{rad}$. The small discrepancy can be attribute to the simplicity of the model, that does not consider any nonlinear effect, but it should also be considered that the piezo actuator position has a $+/-20 \%$ uncertainty at the moment. More accurate measurements intended to verify the accuracy of the model will be performed.

breadboard. The difference between the two out-of-loop f- $2 \mathrm{f}$ phases yields $200 \mathrm{mrad} \mathrm{rms}$ of non-common mode phase, allowing to estimate an upper limit of about $200 \mathrm{mrad} \mathrm{rms}$ for the CEP fluctuations (the common mode detection noise due to common energy, pointing and air fluctuations is still present
The seed pulse, obtained by supercontinuum generation, inherited, to the first order, the shot-to-shot fluctuating phase $\varphi_{\text {pump }}$ from the pump pulse. Moreover, the delay between the seed and the pump pulse can be controlled by a delay line (a 
piezo actuator in our experiment). In the reference frame of the pump this can be written as:

$$
\mathrm{E}_{\text {seed }}(\mathrm{t}-\mathrm{T})=\exp \left(-\mathrm{i}\left(\omega_{\text {seed }}(\mathrm{t}-\mathrm{T})+\varphi_{\text {pump }}\right)\right),
$$

where the seed envelope has been treated as constant since in our experiment it is longer than the pump duration and the strong parametric amplification imprints the pump envelope on the output signal regardless of the initial seed temporal profile. The output signal and idler pulses will then be:

$\mathrm{E}_{\text {signal }}(\mathrm{t})=\alpha \mathrm{A}_{\text {pump }}(\mathrm{t}) \exp \left\{-\mathrm{i}\left[\omega_{\text {seed }}(\mathrm{t}-\mathrm{T})+\varphi_{\text {pump }}\right]\right\}=$

$\alpha A_{\text {pump }}(t) \exp \left\{-\mathrm{i}\left[\omega_{\text {seed }} \mathrm{t}-\left(2 \pi \mathrm{c} / \lambda_{\text {seed }}\right) \mathrm{T}\right]+\varphi_{\text {pump }}\right\}$

$\mathrm{E}_{\text {idler }}(\mathrm{t})=\beta \mathrm{A}_{\text {pump }}(\mathrm{t}) \exp \left\{-\mathrm{i}\left[\omega_{\text {pump }} \mathrm{t}+\varphi_{\text {pump }}-\omega_{\text {seed }}(\mathrm{t}-\mathrm{T})-\varphi_{\text {pump }}\right]\right\}$

$=\beta A_{\text {pump }}(\mathrm{t}) \exp \left[-\mathrm{i}\left(\omega_{\text {idler }} \mathrm{t}+\omega_{\text {seed }} \mathrm{T}\right)\right]$

$=\beta A_{\text {pump }}(\mathrm{t}) \exp \left\{-\mathrm{i}\left[\omega_{\text {idler }} \mathrm{t}+\left(2 \pi \mathrm{c} / \lambda_{\text {seed }}\right) \mathrm{T}\right]\right\}$.

The envelopes of signal and idler pulses are locked by the amplification to the pump envelope, and $\alpha$ and $\beta$ are constants $(<1)$ that accounts for the conversion efficiency.

Equations (1) and (2) show, that if a delay $T=n \lambda_{\text {seed }} / \mathrm{c}$ (n refractive index of air, c vacuum speed of light) is introduced between the seed and the pump pulses, the CEP of both signal and idler pulses is changed by $2 \pi$. However, the CEP of the signal pulse remains unstable, since the $\varphi_{\text {pump }}$ phase changes randomly from pulse to pulse.

In our experiment, we change the pump pulse path length, instead of the seed path length, as in the model above. In this case, we should additionally consider that the pump's envelope and pump's phase propagate at different velocities. However, in air the velocity difference @ $800 \mathrm{~nm}$ is $\mathrm{v}_{\text {phase }}-$ $\mathrm{v}_{\text {group }}=1.5 \times 10^{3} \mathrm{~m} / \mathrm{s}$, that for a maximum delay $\mathrm{T}$ introduced by our piezo of about $15 \mathrm{fs}$, generates an additional CEP shift of less than $4 \times 10^{-5} \pi$, too small to be measured with current technology. The validity of this model is proven by experimental results in figure 3(b).

\section{CONCLUSION}

In conclusion, we have presented a front-end setup capable of generating multiple CEP-stable and CEP-controlled WLs, suitable for seeding a multi-channel $\mathrm{OP}(\mathrm{CP}) \mathrm{A}$ waveform synthesizer. Moreover, the physical process of imprinting the carrier-envelope phase on the idler by pump and seed has been clarified.

\section{REFERENCES}

[1] O. D. Mücke et al., IEEE J. Sel. Top. Quantum Electron. 21, 8700712 (2015)

[2] R.E. Mainz et al., Conference on Lasers and Electro-Optics, OSA Technical Digest (2016) Optical Society of America, paper STu1I.6.

[3] C. Manzoni et al., J. Opt. 18 (2016) 103501 (33pp)

[4] G. Cerullo et al., Laser Photonics Rev. 5, No. 3, 323-351 (2011) 